support and education relating to sexuality and sexual health. Feedback from Momentum participants for the period of February 2014 to April 2015 are collected and analysed.

Results Participants expressed heightened knowledge of their sexuality after attending the workshop. The workshop also provided them with an educational environment relating to sexual health. Additionally, many stated the social benefit of the workshop, as they formed friendships that expand their social support network. Many participants felt supported and connected to the gay community, indicating positive benefits gained from attending the workshop.

Conclusion The needs of gay men who came out later in life must be recognised to assist them overcoming homophobia, construct a supportive social network, and provide sexual health education relevant to their lived experiences to improve their well being.

Disclosure of interest statement The Victorian AIDS Council is funded by the Victorian Department of Health and Human Services. No pharmaceutical grants were received in the delivery of the program.

\section{P11.31 HIV/STI PREVALENCE, SEXUAL RISK, AND DRUG USE AMONG MEN WHO HAVE SEX WITH MEN IN URBAN SETTINGS IN VIET NAM}

DC Thanh*, TD Quang, LA Tuan, NT Ha, HTT Ha, HT Tram, PH Thang, NA Tuan. National Institute of Hygiene and Epidemiology, Ha Noi, Viet Nam

\subsection{6/sextrans-2015-052270.479}

Introduction HIV epidemic is still concentrated in most at risk populations including people who inject drugs (PWID), female sex workers (FSW), and men who have sex with men (MSM) in Viet Nam. This aims to describe HIV/STI prevalence and drug use among MSM in Ha Noi, Hai Phong, and Can Tho, Viet Nam.

Methods Cross-sectional surveys were conducted among MSM in 2013. Biologic and behavioural data were collected with the main objectives of obtaining HIV/STI prevalence, risk and preventive behaviours which focused on sexual and drug-using behaviours and exposure to HIV/AIDS intervention programs.

Results HIV prevalence was 4.0\%, 5.4\%, and 1.1\% in Ha Noi, Hai Phong, and Can Tho, respectively. Chlamydia Trachomatis (CT) was $4.4 \%, 5.0 \%$, and $3.5 \%$ in Ha Noi, Hai Phong, and Can Tho, respectively. Neisseria Gonorrhoea (NG) was 3.4\%, $0.3 \%$, and $3.2 \%$ in rectal and $0.0 \%, 0.3 \%$, and $0.9 \%$ in genital in Ha Noi, Hai Phong, and Can Tho, respectively. Consistently condom use with non-commercial male partners in past month was $59.1 \%, 78.6 \%$, and $62.3 \%$ in Ha Noi, Hai Phong, and Can Tho, respectively. Reported ever drug use was 13.2\%, 13.1\%, and $1.5 \%$ in Ha Noi, Hai Phong, and Can Tho, respectively. Opiate in urinalysis was $5.3 \%, 11.0 \%$, and $1.2 \%$ in Ha Noi, Hai Phong, and Can Tho, respectively. Amphetamine-type stimulants (ATS) positive in urinalysis was $2.8 \%, 0.7 \%$, and $0.0 \%$ in in $\mathrm{Ha}$ Noi, Hai Phong, and Can Tho, respectively.

Conclusion HIV prevalence was relatively high in $\mathrm{Ha}$ Noi and Hai Phong. There was an evidence of unsafe sex since the presence of other STIs. Non-injection drug use, particularly ATS use, among MSM has gained increasing attention. HIV prevention efforts need a refocus on sexual and drug using risk reduction for MSM.

Disclosure of interest None.
P11.32 SOCIO-DEMOGRAPHIC AND BEHAVIOURAL CHARACTERISTICS OF MEN WHO HAVE SEX WITH MEN (MSM) IN THE NETHERLANDS WHO HAVE NEVER BEEN TESTED FOR HIV

C Den Daas, M Doppen, E Op de Coul*. Centre for Infectious Disease Control (RIVM), The Netherlands

\subsection{6/sextrans-2015-052270.480}

Introduction The proportion of MSM unaware of their HIV infection is $31 \%$ in the Netherlands. People who are unaware of HIV are more likely to transmit HIV to others and are unable to benefit from (early) treatment. To improve HIV testing, it is crucial to identify characteristics of MSM who were never tested.

Methods We analysed HIV testing behaviour in the Dutch sample $(\mathrm{n}=3,787)$ of the European MSM Internet Survey (EMIS) in relation to socio-demographic and behavioural factors by logistic regression.

Results Of the MSM, 20.5\% reported to be 'never tested for HIV'. The adjusted model showed that MSM of younger age ( $<25$ yrs aOR 4.6 CI 3.7-5.8 vs $>40$ yrs), with lower education (2.1 CI 1.8-2.4 vs high), with lower HIV knowledge (5.5 CI 3.8-8.0 vs higher) had higher odds on 'never tested for HIV'. Further, MSM with lower sexual risks were more likely to be never tested for HIV; MSM with no UAI $<12$ months (3.8 CI $3.1-4.8$ vs yes), never visiting gay venues (3.9 CI 3.3-4.7 vs ever), with no STIs < 12 months (11.3 CI 6.6-19.3 vs yes), no sex/party drugs (2.7 CI 2.0-3.7 vs yes), outness (3.4 CI 2.9-4.0 vs other), and having more gay friends (few 6.0 CI 4.6-7.9 vs most are gay). Additionally, of all MSM who were never tested for HIV $70.3 \%$ had anal intercourse with $\geq 1$ casual partner (s) $<12$ months and $47 \%$ had unprotected intercourse.

Conclusion MSM with lower sexual risks were more likely to be never tested for HIV, suggesting that MSM make risk assessments to inform their choices about HIV testing. Nevertheless, MSM who never tested for HIV also showed sexual behaviour that put them at HIV risk, and are therefore important to target for HIV interventions.

Disclosure of interest statement The study is funded by the RIVM. No pharmaceutical grants were received in the development of this study.

\section{P12 - STI care}

\section{P12.01 GETTING YOUR CHLAMYDIA CARE ONLINE: QUALITATIVE STUDY AMONG USERS OF THE CHLAMYDIA ONLINE CLINICAL CARE PATHWAY (CHLAMYDIA-OCCP), IN A PROOF OF CONCEPT STUDY}

${ }^{1} \mathrm{CRH}$ Aicken, ${ }^{2} \mathrm{U}$ Sutcliffe, ${ }^{2} \mathrm{CS}$ Estcourt*, ${ }^{2} \mathrm{~J} \mathrm{Gibbs,}{ }^{2} \amalg$ Tickle, ${ }^{1} \mathrm{P}$ Sonnenberg, ${ }^{3} \mathrm{ST}$ Sadiq, ${ }^{1} \mathrm{M}$ Shahmanesh. 'University College London; ${ }^{2}$ Queen Mary University of London; ${ }^{3}$ St George's, University of London

\subsection{6/sextrans-2015-052270.481}

Background Online clinical care was offered to people receiving positive chlamydia results, following testing in Genitourinary Medicine clinics or through six National Chlamydia Screening Programme areas' online postal self-sampling service: 21.07.1413.3.15, in a proof-of-concept study within the eSTI ${ }^{2}$ consortium (www.esti2.org.uk). Chlamydia-OCCP included: STI results service; clinical consultation; electronic prescription via community pharmacy; partner notification; and a telephone helpline 
to support patients and to fast-track those with complex needs to clinic.

We describe views and experiences of people who chose Chlamydia-OCCP for treatment.

Methods An interviewer conducted 40 in-depth follow-up telephone interviews with a purposive sample. Thematic analysis. Results Participants described choosing Chlamydia-OCCP over alternatives for its expected greater speed, convenience and privacy; or trying it out without preconceptions. They valued rapid access to treatment, but also obtaining treatment when convenient.

Despite general concerns about keeping their chlamydia diagnoses secret, several accessed the online consultation immediately, at work/in public. Participants described Chlamydia-OCCP web-app as easy to use, with only minor technical or privacy issues. They found providing personal/sensitive information online acceptable, reasoning that: 1 . it was preferable to (potentially embarrassing) face-to-face consultations; 2 . ChlamydiaOCCP was associated with trusted services; 3 . they already provided personal information online for other services.

Barriers to rapid treatment via Chlamydia-OCCP included: difficulties accessing pharmacy due to employment/carer demands or holiday/business travel (these also impeded clinic access); pharmacy staff's knowledge of process. Collecting treatment from pharmacies was universally acceptable, despite difficulties experienced by some.

Reactions of participants fast-tracked to clinic varied (anxiety, disappointment, relief), and helpline contact reassured them, aiding their understanding of why a clinic visit was necessary.

Conclusion Participants expressed satisfaction with the web-app and helpline, using Chlamydia-OCCP to obtain treatment discreetly, conveniently and quickly, despite busy lifestyles. Further evaluation of the pharmacy process will explore how this can be optimised, to further improve time-to-treatment and satisfaction. Disclosure of interest statement Nothing to declare.

\section{P12.02 DEVELOPING AND USING THE ECLINICAL CARE PATHWAY FRAMEWORK: A NOVEL TOOL FOR CREATING ONLINE CLINICAL CARE PATHWAYS AND ITS APPLICATION TO MANAGEMENT OF GENITAL CHLAMYDIA}

1J Gibbs*, 'U Sutcliffe, ${ }^{2} V$ Gkatzidou, ${ }^{3} \mathrm{P}$ Sonnenberg, ${ }^{2} \mathrm{~K}$ Hone, ${ }^{1} \mathrm{R}$ Ashcroft, ${ }^{4} \mathrm{E}$ HardingEsch, ${ }^{4} \mathrm{C}$ Lowndes, ${ }^{5} \mathrm{ST}$ Sadiq, ${ }^{1} \mathrm{CS}$ Estcourt. 'Queen Mary University of London; ${ }^{2}$ Brunel University London; ${ }^{3}$ University College London; ${ }^{4}$ Public Health UK; ${ }^{5}$ St George's University of London

\subsection{6/sextrans-2015-052270.482}

Introduction Home sexually transmitted infection (STI) sampling and remote STI diagnostics create potential for online treatment. Currently there is no guidance on development or content of online clinical consultations and, particularly, no recommendations relevant to sexual health. We describe creation of a new framework for developing complex online clinical care pathways and its application to management of people with genital chlamydia.

Methods We developed the eClinical Care Pathway Framework (eCCPF) consisting of a nine-step iterative process, Step 1: determines aims of the online clinical care pathway; Step 2: defines the functional units of the pathway; Step 3: drafts the online clinical consultation; Step 4: expert review; Step 5: comprehension testing; Step 6: user-centred interface testing; Step 7: specification development; Step 8: usability testing and further comprehension testing; Step 9: pilots the pathway. We then applied the framework to create the Chlamydia Online Clinical Care Pathway (Chlamydia-OCCP).

Results Using the eCCPF enabled us to elucidate the different sequence of functional units of care, as in contrast to traditional medicine, the Chlamydia-OCCP starts with provision of test results (diagnosis). Users then obtain infection-related information, before completing an online automated clinical consultation (medical and sexual history, partner notification), before collection of antibiotics from a community pharmacy. This enables a more focussed approach to assessment of safety of antibiotic prescribing than lengthier traditional medication history questions.

Conclusion By following each step of the eCCPF, the resulting Chlamydia-OCCP has a different sequence to traditional care pathways and is adapted to the needs of remote testing and online care. It provides the clinical services and surveillance functions required to meet UK national standards. This standardised method of collecting data on demography and sexual behaviour, with easily extractable data and potential for interoperability with surveillance systems, could be a powerful tool for public health and clinical management.

Disclosure of interest statement Nothing to declare.

\section{P12.03 HOW ACCURATE AND COMPREHENSIVE ARE CURRENTLY AVAILABLE MOBILE MEDICAL APPLICATIONS (APPS) FOR SEXUALLY TRANSMITTED AND GENITAL INFECTIONS: A COMPREHENSIVE REVIEW}

'1 Gibbs*, ${ }^{2} V$ Gkatzidou, 'L Tickle, 'SR Manning, ${ }^{1} T$ Tilakkumar, ${ }^{2} \mathrm{~K}$ Hone, ${ }^{1}$ RE Ashcroft, ${ }^{3} \mathrm{P}$ Sonnenberg, ${ }^{4} \mathrm{ST}$ Sadiq, ${ }^{1} \mathrm{CS}$ Estcourt. 'Queen Mary University of London; ${ }^{2}$ Brunel University of London; ${ }^{3}$ University College London; ${ }^{4}$ St George's University of London

\subsection{6/sextrans-2015-052270.483}

Introduction In the UK, $88 \%$ of young people, a group at high risk of STIs, own a smartphone. Seeking sexual health advice online is common and provision of STI apps is increasing. The UK lacks a robust framework for quality control of mobile medical apps and potential for misinformation is substantial. We undertook a comprehensive review of content and accuracy of contemporary apps for STIs (except HIV) and related genital infections aimed at members of the public.

Methods Search of Googleplay and iTunes stores for free and paid apps regarding STIs and genital infections, testing, diagnosis and management, between 10/09/2014-16/09/2014. We assessed eligible apps against: 1) 19 modified Health on The Net (HON) Foundation principles; 2) comprehensiveness and accuracy of information on individual STIs/genital infections, and their diagnosis and management, compared with respective National Health Service STI information webpage content.

Results 144/6642 apps met eligibility criteria (Android $n=94$; iOS $n=29$; both $n=24)$. 57 were excluded after downloading. 87 apps (52 Android, 20 iOS, 15 both) were analysed. Only $29 \%$ apps met $>/=6 \mathrm{HON}$ criteria. Content was highly variable: $34 / 87$ (39\%) covered one or two infections; 16/34 (47\%) were ebooks, predominately about genital herpes or candidiasis; 40/87 (46\%) covered multiple STIs; $5 / 87$ (6\%), solely focused on accessing STI testing. In terms of accuracy: 13/87 (15\%) were fully, 46/87 (53\%) mostly and 28/87 (32\%) partially accurate. $25 / 87(29 \%)$ contained $>/=1$ instance of potentially harmful information. Apps available on both platforms had a greater degree of accuracy than single platform apps. Only 1 app 